\title{
Characterization of QTLs for Root Traits of Wheat Grown under Different Nitrogen and Phosphorus Supply Levels
}

\author{
Yongzhe Ren ${ }^{1 * t}$, Yingying Qian ${ }^{2 t}$, Yanhua Xu ${ }^{1}$, ChunQin Zou ${ }^{2}$, Dongcheng Liu ${ }^{3}$, \\ Xueqiang Zhao ${ }^{3 *}$, Aimin Zhang ${ }^{3}$ and Yiping Tong ${ }^{3}$ \\ ${ }^{1}$ State Key Laboratory of Wheat and Maize Crop Science, College of Agronomy, Collaborative Innovation Center of Henan \\ Grain Crops, Henan Agricultural University, Zhengzhou, China, ${ }^{2}$ China Agricultural University, Beijing, China, ${ }^{3}$ State Key \\ Laboratory for Plant Cell and Chromosome Engineering, Institute of Genetics and Developmental Sciences, Chinese \\ Academy of Sciences, Beijing, China
}

OPEN ACCESS

Edited by:

Hatem Rouached,

Institut National de la Recherche

Agronomique (INRA), France

Reviewed by:

Peter Ryan,

Commonwealth Scientific and Industrial Research Organisation

(CSIRO), Australia

Ajay Kumar Pandey,

National Agri-Food Biotechnology

Institute, India

*Correspondence:

Xueqiang Zhao

xqzhao@genetics.ac.cn Yongzhe Ren

yongzheren66@163.com

${ }^{\dagger}$ These authors have contributed equally to this work.

Specialty section:

This article was submitted to

Plant Nutrition,

a section of the journa

Frontiers in Plant Science

Received: 06 August 2017 Accepted: 24 November 2017 Published: 11 December 2017

Citation:

Ren Y, Qian Y, Xu Y, Zou C, Liu D, Zhao X, Zhang A and Tong Y (2017) Characterization of QTLs for Root Traits of Wheat Grown under Different

Nitrogen and Phosphorus Supply

Levels. Front. Plant Sci. 8:2096.

doi: 10.3389/fp/s.2017.02096
Root is important in acquiring nutrients from soils. Developing marker-assisted selection for wheat root traits can help wheat breeders to select roots desirable for efficient acquisition of nutrients. A recombinant inbred line (RIL) population derived from wheat varieties Xiaoyan 54 and Jing 411 was used to detect QTLs for maximum root length and root dry weight (RDW) under control, low nitrogen and low phosphorus conditions in hydrophobic culture $(\mathrm{HC})$. We totally detected 17 QTLs for the investigated root traits located at 13 loci on 11 chromosomes. These loci differentially expressed under different nutrient supplying levels. The RILs simultaneously harboring positive alleles or negative alleles of the most significant three QTLs for RDW, qRDW.CK-2A, qRDW.CK-2D, and qRDW.CK-3B, were selected for soil column culture (SC) trial to verify the effects of these QTLs under soil conditions. The RILs pyramiding the positive alleles not only had significantly higher shoot dry weight, RDW, nitrogen and phosphorus uptake in all the three treatments of the $\mathrm{HC}$ trial, but also had significantly higher RDW distribution in both the top- and sub-soils in the SC trial than those pyramiding the negative alleles. These results suggested that QTL analysis based on hydroponic culture can provide useful information for molecular design of wheat with large and deep root system.

Keywords: Triticum aestivum L., quantitative trait locus (QTL), root traits, nitrogen deficiency, phosphorus deficiency

\section{INTRODUCTION}

High crop yield largely depends on input of water and fertilizers. During 1960-2000 of the last century, the world food production nearly doubled, but the annual consumption of nitrogen $(\mathrm{N})$ and phosphorus (P) fertilizers increased 7.8-folds (from 10 to $88 \mathrm{Tg}$ ) and 3.4-folds (from 9 to $40 \mathrm{Tg}$ ), respectively; and the irrigated land increased from 10 to $18 \%$ of arable land (Vance, 2001). The high input has led to degradation of soil, air and water quality, and the exhaustion of natural resources such as water and nutrients (Holford, 1997; Vance et al., 2003; Guo et al., 2010). Moreover, producing more food for the growing population will put further enormous pressure on natural resources and environments. Therefore, it is needed to improve resource use efficiency of crops in the future to minimize the negative impact of increasing crop yield on natural resources and environments. 
In the past, the development of high-yield varieties of cereal crops were mostly achieved by selection of the aboveground organs (e.g., the first green revolution), and the belowground roots were largely neglected (Waines and Ehdaie, 2007). Considering the importance of roots in determining acquisition efficiency of soil resources like nutrients and water, manipulating root traits such as RSA and nutrient uptake has been suggested to enable a very much needed new green revolution and further increase in yields (de Dorlodot et al., 2007; Lynch, 2007; Den Herder et al., 2010). However, roots, the hidden half of a plant, are difficult to be selected directly by breeders, thus developing marker-assisted selection (MAS) will help breeders to select root traits desirable for efficient acquisition of water and nutrients from soils. QTL analysis has observed overlap between QTLs for root traits and those for nutrient uptake and productivity in many crop species such as wheat (Sharma et al., 2011; Xie et al., 2017; Yuan et al., 2017), rice (Steele et al., 2007; Uga et al., 2013), maize (Tuberosa et al., 2002; Hochholdinger and Tuberosa, 2009), and soybean (Liang et al., 2010), suggesting the valuable potential of marker-assisted breeding for root traits in increasing resource use efficiency and yields.

Wheat is one of the most important food crops in the world; thus identifying QTLs toward marker-assisted breeding for root traits desirable for efficient acquisition of soil nutrients may offer a sustainable solution to nutrient management in wheat production. It is well known that root development is susceptible to nutrient availability, therefore understanding genetic basis of roots responding to nutrient availability is of significance in breeding wheat with root system desirable for nutrient uptake in a wide nutrient regime. A number of literatures have reported QTLs for root biomass and morphological parameters in wheat (Laperche et al., 2006; Sanguineti et al., 2007; Canè et al., 2014; Atkinson et al., 2015; Maccaferri et al., 2016; Iannucci et al., 2017). However, the most challenge in identifying QTLs for root traits is the difficulty in observing roots grown in the soils, especially when analyzing a large number of genotypes in QTL mapping. Up to now, root QTLs were chiefly detected by using hydroponic culture or sand culture. Although QTLs for root characteristics detected in hydroponics have been reported to coincide with nutrient uptake and yield components by wheat (An et al., 2006; Canè et al., 2014; Horn et al., 2016) in field trials, whether QTLs expressed in hydroponics can be used in selecting root traits of soil-grown wheat plants is still uncertain.

In order to clarify this problem, we designed the following experiments. Firstly, we mapped QTLs for root traits and analyzed their relations with nitrogen $(\mathrm{N})$ and phosphorus $(\mathrm{P})$ uptake under different nitrogen and phosphorus supply levels in a hydroponic culture using a recombinant inbred line (RIL) population. Furthermore, the RILs simultaneously harboring positive alleles (8 lines) or negative alleles (11 lines) of the top three QTLs for RDW in phenotypic contribution rate were selected for soil culture (SC) trial to verify the effects of these QTLs under soil conditions. The results showed that QTL analysis based on hydroponic culture can provide practical information for molecular design of wheat with large and deep root system and efficient nutrient uptake.

\section{MATERIALS AND METHODS}

\section{Plant Materials}

One recombinant inbred line (RIL) population was used in this study. The RIL population contained 142 RILs derived from two Chinese winter wheat varieties Xiaoyan 54 and Jing 411. Xiaoyan 54 has been shown to have higher efficiency in acquisition of nitrogen from soil than Jing 411, regardless of the soil depth, consistent with the finding that Xiaoyan 54 had a bigger and deeper root system than Jing 411 (Zhang et al., 2005).

\section{Root Morphology Evaluation}

A hydroponic culture (HC) experiment was carried out to phenotype the RIL population at seedling stage. In the hydroponic culture, seeds were germinated in saturated $\mathrm{CaSO}_{4}$ solution for 7 days at $20^{\circ} \mathrm{C}$, and then the germinated seeds with residual endosperm removed were transferred to three tanks $(2.5 \mathrm{~m}$ long $\times 1.5 \mathrm{~m}$ wide $\times 0.5 \mathrm{~m}$ high $)$ each containing $1,800 \mathrm{~L}$ of nutrient solution as described previously (Li et al., 2011; Figure S1). The tanks contained sufficient full strength $(\mathrm{CK})$, low nitrogen $(\mathrm{N})$, and low phosphorus (P) nutrient solution, respectively. The $\mathrm{CK}$ nutrient solution contained $(\mathrm{mM}) \mathrm{Ca}\left(\mathrm{NO}_{3}\right)_{2} 1, \mathrm{KH}_{2} \mathrm{PO}_{4} 0.2, \mathrm{MgSO}_{4} 1, \mathrm{KCl} 1.5, \mathrm{CaCl}_{2}$ $1.5, \mathrm{H}_{3} \mathrm{BO}_{3} 1 \times 10^{-3},\left(\mathrm{NH}_{4}\right)_{6} \mathrm{Mo}_{7} \mathrm{O}_{24} 5 \times 10^{-5}, \mathrm{CuSO}_{4} 5 \times 10^{-4}$, ZnSO4 $1 \times 10^{-3}$, MnSO4 $1 \times 10^{-3}, \mathrm{Fe}(\mathrm{III})-\mathrm{EDTA} 1 \times 10^{-1}$. The low $\mathrm{N}$ (LN) nutrient solution contained the same nutrients concentration with full strength nutrient solution except $\mathrm{Ca}\left(\mathrm{NO}_{3}\right)_{2} \quad(0.1 \mathrm{mM})$ and $\mathrm{CaCl}_{2}(2.4 \mathrm{mM})$. The low $\mathrm{P}$ (LP) nutrient solution contained the same nutrients concentration with full strength nutrient solution except $\mathrm{KH}_{2} \mathrm{PO}_{4}(0.01 \mathrm{mM})$ and $\mathrm{KCl}(1.69 \mathrm{mM})$. The $\mathrm{pH}$ of nutrition solution was adjusted to 6.0 with dilute $\mathrm{HCl}$ and $\mathrm{NaOH}$ before transferring. The RILs were randomly placed and grown in a greenhouse with three replications each. About 450 plants were grown in each tank. The solution was changed every 7 days. The lowest temperature of the greenhouse ranged from 5 to $10^{\circ} \mathrm{C}$, and the highest temperature varied from 18 to $30^{\circ} \mathrm{C}$ of each day during the period of HC trial. The root morphologies and other related traits of the "Xiaoyan $54 \times$ Jing 411 " RIL population were investigated after these lines were grown for 35 days in the hydroponic trial. The developmental stages of the parents of the RIL population, Xiaoyan54 and J411, were Zadoks growth scale 24 and 23, respectively under CK condition; while the developmental stage of Xiaoyan54 and J411 were ranged from Zadoks growth scale 21 to 22 under LN and LP conditions (Zadoks et al., 1974).

To verify whether QTLs expressed in hydroponics can be used in selecting root traits of soil-grown wheat plants, The RILs simultaneously harboring positive ( 8 lines) or negative alleles (11 lines) of the top three QTLs for RDW in phenotypic contribution rate were selected as materials to conduct a soil column culture (SC) experiment to phenotype the root traits and nutrient $(\mathrm{N}$, $\mathrm{P}$ ) use efficiency under soil culture condition. The SC trial was carried out in the experimental station of Institute of Genetics and Developmental Biology, Chinese Academy of Sciences in Beijing. Before sowing, $87.8 \mathrm{mg} \mathrm{KH}_{2} \mathrm{PO}_{4}, 107.14 \mathrm{mg}$ urea and $4.4 \mathrm{mg} \mathrm{ZnSO}_{4} .7 \mathrm{H}_{2} \mathrm{O}$ were well mixed with every 1 kilogram soil used in this trial. Seeds were germinated for $24 \mathrm{~h}$ at $20^{\circ} \mathrm{C}$, and 
then five germinated seeds were sowed in each PVC tube with $90 \mathrm{~cm}$-high and $11 \mathrm{~cm}$-diameter. Wheat plants were thinned to three plants per tube at the 2nd leaf developmental stage (Zadoks growth scale 12) (Zadoks et al., 1974). The sowing date was September 27, 2014, which was the suitable time for winter wheat sowing in field in Beijing. The soil columns were randomly placed and with three replications each. The lowest temperature ranged from 5 to $12^{\circ} \mathrm{C}$, and the highest temperature varied from 15 to $27^{\circ} \mathrm{C}$ of each day during the period of SC trial. Plants were harvested 35 days after germination. The roots were washed free of sand to evaluate root traits.

In the hydroponic culture trial, the maximal root length (MRL), root dry weight (RDW), tiller (TN), shoot dry weight (SDW), were investigated under sufficient nutrient supply, low $\mathrm{N}$ and low $\mathrm{P}$ conditions; while in the soil column trial, the root dry weight distribution in the soil profiles $0-30 \mathrm{~cm}$ (RDW1), 30$60 \mathrm{~cm}$ (RDW2), $>60 \mathrm{~cm}$ (RDW3), TN, SDW were investigated under sufficient nutrient condition. Total $\mathrm{N}$ and $\mathrm{P}$ concentration in root and shoot tissues were measured according to previous reported literatures (An et al., 2006; Su et al., 2006) in both trials. The N (NUP) and P (PUP) uptake were calculated as the sum of $\mathrm{N}$ or $\mathrm{P}$ accumulated in root $(\mathrm{RDW} \times \operatorname{root} \mathrm{N}(\mathrm{P})$ concentration) and shoot (SDW $\times$ Shoot $\mathrm{N}(\mathrm{P})$ concentration).

\section{Statistical Analysis}

The means, standard deviations, standard errors and ranges of each measured morphological traits were calculated by using SPSS11.5 software. The statistical significance of differences across the RILs and the two parents were analyzed by using Fischer LSD (least-significant difference) test for all of the traits, with $P$-value $<0.05$ considered significant. The correlations between different traits were also analyzed by using SPSS11.5 software.

\section{QTL Detection}

The genetic map of the "Xiaoyan $54 \times$ Jing 411 " RIL population was described by Ren et al. (2012). The composite interval mapping was applied for QTL mapping (Zeng, 1994). In the forward regression analysis, the walk speed and window size were set as 2 and $10 \mathrm{cM}$, respectively, with five control markers. The phenotypic variation explained by a single QTL was determined by the square of the partial correlation coefficient $\left(R^{2}\right)$. Analyses of QTL location, additive effect and 95\% confidence intervals of QTLs were performed using WinQTLCart 2.5 software (Model 6, Basten et al., 2001). The threshold of LOD value for QTL detection was set as 2.5 .

\section{RESULTS}

\section{Evaluation of Phenotypes}

We evaluated RDW and MRL under CK, low $\mathrm{N}$ and low $\mathrm{P}$ conditions. As shown in Table 1, the female parent Xiaoyan 54 had similar RDW and MRL with the male parent Jing 411 under CK condition, but it had higher RDW and longer MRL than Jing 411 under both low $\mathrm{N}$ and low $\mathrm{P}$ conditions. Low $\mathrm{N}$ and low $\mathrm{P}$ treatments increased RDW and MRL of the female parent Xiaoyan 54, but did not significantly influenced on that of Jing 411 (except that low N reduced MRL in Jing 411) (Table 1). The differential response of RDW and MRL to low $\mathrm{N}$ and low $\mathrm{P}$ stresses also existed among the RILs (Table S1). The RIL lines showed large variations in all the investigated traits (Table 1). There existed RILs with values that were higher or lower than both parents in all the evaluated traits, indicating potential transgressive variations and the presence of positive and negative alleles in both parents.

We also investigate the above-ground traits (TN and SDW) and nutrient uptake (NUP and PUP). These four traits exhibited large variations among the RIL lines (Table S2). There were significantly and positively correlation coefficients between $\mathrm{TN}$, SDW, RDW NUP and PUP in all the three treatments (Table S3), indicating that wheat lines with vigorous roots and shoots could facilitate $\mathrm{N}$ and $\mathrm{P}$ uptake. Generally, MRL showed relative poorer correlations with other investigated traits (Table S3).

\section{Identification of QTLs for Root Traits}

The trial detected eight QTLs for total root dry weight (RDW) and nine QTLs for maximum root length (MRL), respectively. These 17 QTLs located at 13 loci on 11 chromosomes (Figure 1). The locus on chromosomes 2D (Xgwm539-Xgwm157) controlled RDW under all the three treatments, the locus on chromosomes 2B (Xgwm210-Xbarc1138.2) associated with MRL under all the

TABLE 1 | Mean values and ranges for root traits (RDW and MRL) in the RIL population and their parents under CK, low N and low $\mathrm{P}$ conditions.

\begin{tabular}{|c|c|c|c|c|c|c|}
\hline \multirow[t]{2}{*}{ Trait } & \multirow[t]{2}{*}{ Treatment } & \multicolumn{2}{|c|}{ Parent (Mean \pm SE) } & \multicolumn{3}{|c|}{ RIL } \\
\hline & & Xiaoyan54 & Jing 411 & Mean \pm SD & Min. & Max. \\
\hline \multirow[t]{3}{*}{ RDW } & CK & $0.071 \pm 0.020$ & $0.078 \pm 0.010$ & $0.067 \pm 0.019$ & 0.030 & 0.128 \\
\hline & Low N & $0.107 \pm 0.011(\mathrm{~A})$ & $0.084 \pm 0.008(B)$ & $0.109 \pm 0.027$ & 0.056 & 0.208 \\
\hline & Low P & $0.121 \pm 0.014(\mathrm{~A})$ & $0.083 \pm 0.001(\mathrm{~B})$ & $0.092 \pm 0.021$ & 0.044 & 0.140 \\
\hline \multirow[t]{3}{*}{$\mathrm{MRL}$} & CK & $41.3 \pm 4.4$ & $39.3 \pm 3.7$ & $36.7 \pm 8.7$ & 14.7 & 55.0 \\
\hline & Low N & $54.3 \pm 2.4(\mathrm{~A})$ & $29.7 \pm 7.2(\mathrm{~B})$ & $42.1 \pm 10.0$ & 16.0 & 65.3 \\
\hline & Low P & $51.5 \pm 0.4(A)$ & $38.7 \pm 5.8(B)$ & $38.6 \pm 10.9$ & 18.3 & 64.0 \\
\hline
\end{tabular}

RDW, root dry weight (g plant ${ }^{-1}$ ); MRL, maximum root length (cm).

Statistical difference between the two parents is indicated by different letters after the means. Capital letters designate significance at $P<0.01$. 

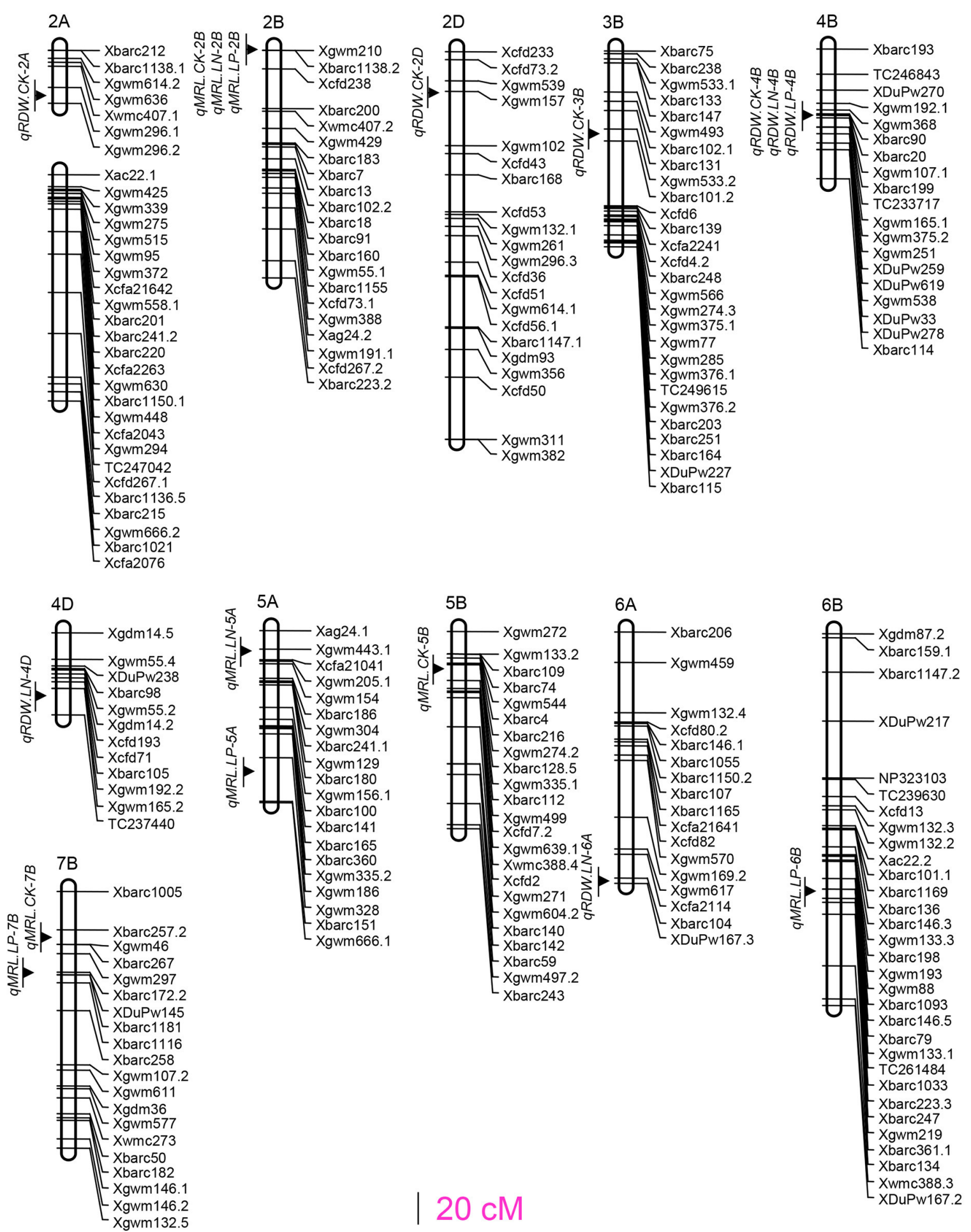

FIGURE 1 | QTLs for the investigated root traits under $\mathrm{CK}$, low $\mathrm{N}$ and low $\mathrm{P}$ conditions.

three treatments (Figure 1; Table 2), and one locus (Xbarc257.2 and Xbarc1116 on chromosome 7B) controlled MRL under CK and low $\mathrm{P}$ conditions. Four loci ( $q R D W . C K-2 A, q R D W . C K-2 D$, $q R D W . C K-3 B$, and $q M R L . C K-5 B)$ were detected to regulate root traits under control condition specifically, three loci ( $q R D W . L N$ $4 D$, $q M R L . L N-5 A$, and $q R D W . L N-6 A$ ) were specifically for root 
TABLE 2 | Detected QTLs for root traits under CK, low N and low P conditions.

\begin{tabular}{|c|c|c|c|c|c|c|c|}
\hline Trait & Treatment & QTL & Chra & Marker interval $^{\mathbf{b}}$ & LODC $^{c}$ & $R^{2} \times 100$ & Additive $^{\mathrm{d}}$ \\
\hline \multirow[t]{8}{*}{ RDW (g/plant) } & CK & qRDW.CK-2A & $2 \mathrm{~A}$ & Xgwm296.1-Xgwm296.2 & 2.7 & 8.9 & -0.006 \\
\hline & CK & $q R D W . C K-2 D$ & $2 \mathrm{D}$ & Xgwm157-Xgwm102 & 3.8 & 15.3 & 0.008 \\
\hline & CK & qRDW.CK-3B & $3 B$ & $\underline{X g w m 533.2-X b a r c 101.2}$ & 3.3 & 10.9 & -0.006 \\
\hline & CK & $q R D W . C K-4 B$ & $4 \mathrm{~B}$ & $\underline{\text { Xbarc90-Xbarc20 }}$ & 3.4 & 7.9 & -0.006 \\
\hline & Low N & qRDW.LN-4B & $4 \mathrm{~B}$ & $\underline{\text { Xbarc } 90-\text { Xbarc20 }}$ & 4.7 & 10.4 & -0.009 \\
\hline & Low N & qRDW.LN-4D & $4 \mathrm{D}$ & Xgwm165.2- TC237440 & 2.9 & 8.8 & 0.008 \\
\hline & Low N & $q R D W . L N-6 A$ & $6 \mathrm{~A}$ & Xbarc104-Xdwpw $\underline{167.3}$ & 3.0 & 8.6 & -0.008 \\
\hline & Low $P$ & $q R D W . L P-4 B$ & $4 \mathrm{~B}$ & TC233717-Xgwm165.1 & 4.1 & 9.6 & -0.007 \\
\hline \multirow[t]{9}{*}{ MRL (cm) } & CK & qMRL.CK-2B & $2 \mathrm{~B}$ & $\underline{X g w m 210-X b a r c 1138.2}$ & 12.8 & 32.7 & 5.1 \\
\hline & CK & qMRL.CK-5B & $5 B$ & Xbarc112-Xgwm499 & 3.5 & 6.5 & -2.3 \\
\hline & CK & qMRL.CK-7B & $7 \mathrm{~B}$ & Xbarc257.2-Xgwm46 & 3.8 & 10.4 & 2.9 \\
\hline & Low N & $q M R L . L N-2 B$ & $2 \mathrm{~B}$ & $\underline{X g w m 210-X b a r c 1138.2}$ & 6.5 & 21.6 & 4.7 \\
\hline & Low N & $q M R L . L N-5 A$ & $5 A$ & Xgwm443.1-Xcfa21041 & 2.7 & 6.8 & -2.7 \\
\hline & Low P & qMRL.LP-2B & $2 \mathrm{~B}$ & $\underline{X g w m 210-X b a r c 1138.2}$ & 5.4 & 16.4 & 4.5 \\
\hline & Low P & $q M R L . L P-5 A$ & $5 A$ & Xgwm328-Xbarc151 & 3.2 & 15.2 & -4.3 \\
\hline & Low P & qMRL.LP-6B & $6 \mathrm{~B}$ & 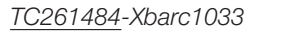 & 2.5 & 5.4 & 2.6 \\
\hline & Low P & qMRL.LP-7B & $7 \mathrm{~B}$ & Xbarc1181-Xbarc1116 & 3.5 & 7.7 & 3.2 \\
\hline
\end{tabular}

a Chr means chromosome name.

${ }^{b}$ Markers underlined were the nearest marker to the QTL.

c LOD means Logarithm of odds.

${ }^{a}$ Additive effects, a positive sign means that positive allele comes from the parent Xiaoyan 54, while a negative sign means positive allele comes from the parent Jing 411.

traits under low $\mathrm{N}$ condition, two loci ( $q M R L . L P-5 A$ and $q M R L . L P-6 B)$ were specifically expressed under low $\mathrm{P}$ condition (Figure 1; Table 2).

\section{Effects of Pyramiding QTLs on RDW and Nutrient Uptake}

We then analyzed the effects of pyramiding QTLs on RDW and nutrient uptake. We analyzed the effects of pyramiding the QTLs for RDW detected under CK and LN conditions, respectively. Firstly, we pyramided the three most significant QTLs of the five QTLs for RDW detected under CK condition ( $q R D W . H C . C K-$ $2 A, q R D W . C K-2 D$, and $q R D W . C K-3 B$ ). The positive pyramiding (the RILs simultaneously harboring the positive alleles at these three QTLs) averagely had 81.0, 64.4, and 55.5\% higher RDW than the negative pyramiding (the RILs simultaneously harboring the negative alleles) under CK, LN and LP conditions, respectively (Table 3 ). Moreover, the positive pyramiding QTLs for RDW significantly increased SDW, NUP and PUP in all the treatments (Table 3). Similarly, the positive pyramiding of the three QTLs for RDW detected under LN condition, $q R D W . L N-4 B, q R D W . L N-4 D$, and $q R D W . L N-6 A$, averagely had $42.3,46.7$, and $29.6 \%$ higher RDW than the negative pyramiding under CK, LN and LP conditions, respectively (Table S4), and increased SDW, NUP, and PUP significantly in all the treatments (Table S4).

To verify the effects of these QTLs under soil conditions, lines simultaneously harboring the positive alleles or negative alleles of the top three QTLs for RDW detected under CK condition were selected for soil culture trial. Also, the positive pyramiding averagely had significantly higher total RDW and root dry weight at different soil layers, and higher nutrient uptake than the negative pyramiding in the SC trial (Table 3 ).

\section{DISCUSSION}

The current study was carried out to detect QTLs for root growth under different $\mathrm{N}$ and $\mathrm{P}$ supply levels. The "Xiaoyan $54 \times$ Jing 411 " RIL population was used in the present study as it has been shown to segregate in root morphology (Ren et al., 2012). Although Xiaoyan 54 and Jing 411 showed no differences in RDW and MRL under CK condition in the HC trial, Xiaoyan 54 had significant higher RDW and longer MRL than Jing 411 under both low $\mathrm{N}$ and low $\mathrm{P}$ conditions (Table 1), indicating the root growth of Xiaoyan 54 more strongly responded to $\mathrm{N}$ - and P-deficiency than that of Jing 411.

Our current study observed large differences among the RILs of the "Xiaoyan $54 \times$ Jing 411" RIL population of all the investigated root traits (Table 1). Both RDW and MRL showed positively and significantly correlations with NUP and PUP in all the treatments (Table S3). These results demonstrated the importance of enhancing root development in improving $\mathrm{N}$ and $\mathrm{P}$ use efficiencies whatever under low $\mathrm{N}$, low $\mathrm{P}$ or $\mathrm{CK}$ condition, confirming the previously reported results that early vigorous growth improves nitrogen and phosphate uptake in wheat (Liao et al., 2004; Ehdaie et al., 2010; Ryan et al., 2015; Wang et al., 2016).

We totally detected 17 QTLs for the investigated root traits located at 13 loci on 11 chromosomes (Figure 1). Pyramiding 
TABLE 3 | Pyramiding QTLs for root biomass enhanced N and P uptake and TGW.

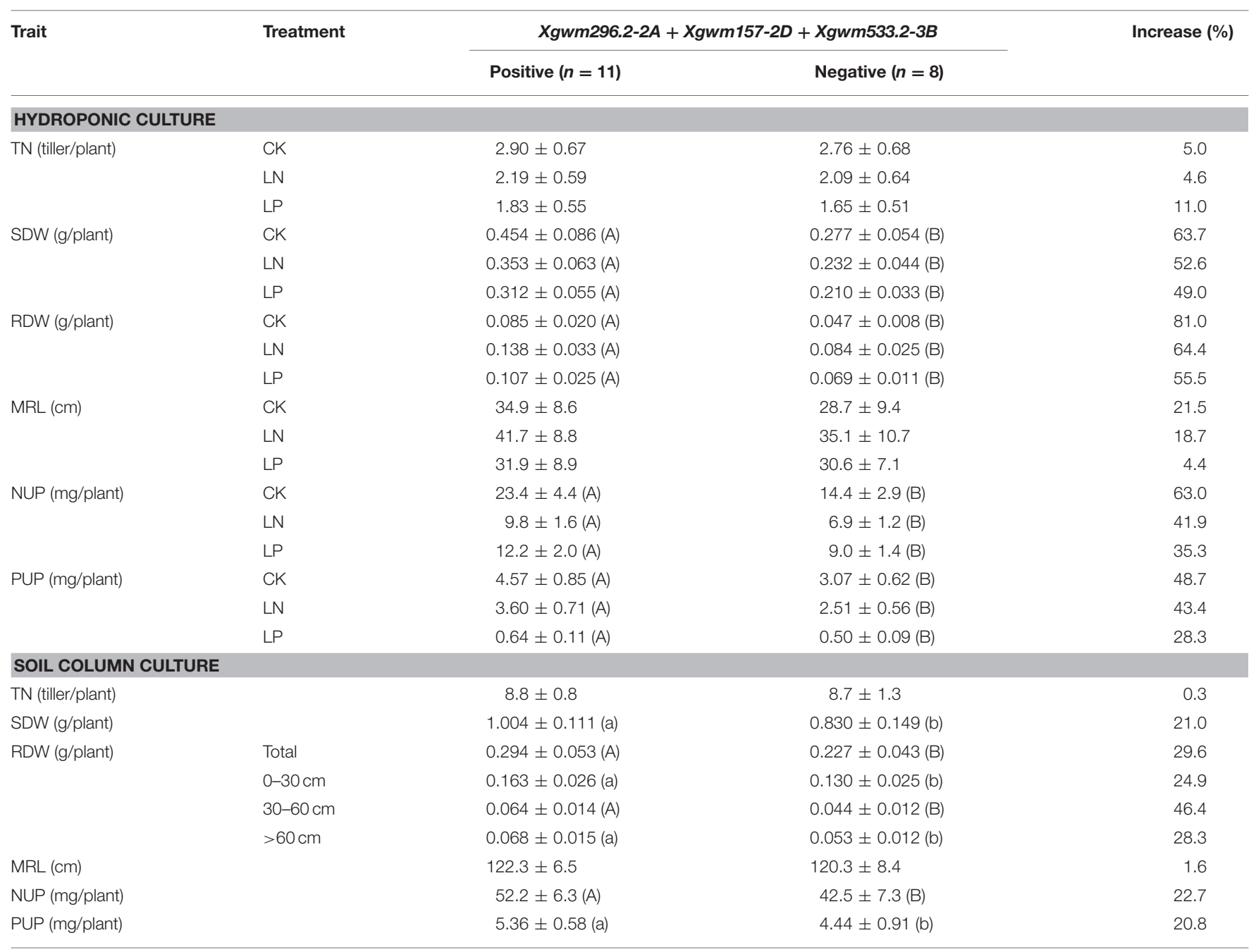

Statistical difference between the positively and the negatively pyramiding groups is indicated by different letters after the means. Capital and small letters designate significance at $P<$ 0.01 and $P<0.05$, respectively.

QTLs for RDW detected in the HC trial not only largely increased RDW, NUP and PUP in the HC trial (Table 3; Table S4), but also significantly increased total RDW and root distribution in both the top- and sup-soils, and consequently enhanced nutrient uptake and shoots growth in the SC trial (Table 3). This result suggested that QTL analysis based on hydroponic culture can provide practical information for molecular design of wheat with large and deep root system. In fact, these three QTLs ( $q R D W . C K$ $2 A, q R D W . C K-2 D$, and $q R D W . C K-3 B$ ) associated with QTLs for root growth or nutrient uptake detected in the current study and reported previously. The QTL $q R D W . C K-2 A$ linked with qSDW.LP-2A, qNUP.LP-2A and qPUP.LP-2A (Figure S2) and a QTL for $\mathrm{P}$ uptake in a P-deficient soil at seedling stage (Su et al., 2006). The QTL $q R D W . C K-2 D$ coincided with seven QTLs for RDW, SDW, NUP, and PUP (Figure S2; Table S5), and RDW at seedling stage, NUP and PUP investigated at seedling stage and maturity in different $\mathrm{N}$ and $\mathrm{P}$ availabilities (An et al., 2006; Su et al., 2009). The QTL $q R D W . C K-3 B$ coincided with QTLs for
SDW under CK and low N conditions, NUP and PUP under CK condition (Figure S2; Table S5), and associated with QTLs for SDW and PUP investigated at seedling stage and maturity in Pdeficient or sufficient soil (Su et al., 2009). The QTLs qRDW.CK$2 A$ and $q R D W . C K-2 D$ also linked with N-use efficient meta-QTL1 and -2 , respectively (Quraishi et al., 2011). These results further supported the importance of these three loci in breeding wheat with improved root growth and nutrient uptake.

Other loci for root growth mapped in the present study also show potential in breeding root growth and nutrient uptake. The QTLs $q R D W . C K-4 B, q R D W . L N-4 B$, and $q R D W . L P-4 B$ were located on the adjacent chromosome region and coincided with a number of QTLs for SDW, PUP and NUP under CK, low N and low $\mathrm{P}$ conditions (Figure S2; Table S5). In previous studies using "Xiaoyan $54 \times$ Jing 411" RIL population, this chromosomal region was identified to affect plant height, harvest index under $\mathrm{CK}$, low $\mathrm{N}$ and low $\mathrm{P}$ conditions, and shoot $\mathrm{N}$ content under low $\mathrm{N}$ condition at adult stages in field trials (Xu et al., 2014), 
and shoot height and biomass production at the seedling stage in both salt stress and control treatments (Xu et al., 2012). In this study, they were also detected to have pleiotropic effects for RDW, SDW, NUP, and PUP traits. Actually, this chromosomal region has been reported controlling RDW and SDW under $\mathrm{CK}$ condition, and biomass under Low $\mathrm{P}$ condition in previous published literatures (Ryan et al., 2015; Maccaferri et al., 2016; Iannucci et al., 2017), and is coincident with that of the Rht$B 1$ gene, which is the main locus involved in the control of plant height (Börner et al., 1996). It was not clear whether $R h t-B 1$ gene to be separated from the QTL for RDW or not. Importantly, this chromosomal region was identified controlling both shoot and root traits at seedling and adult stages, and across various environments, which further confirmed that it has nonenvironment-specific and non-stage-specific genes ( $\mathrm{Xu}$ et al., 2014).

On chromosome 2B, we mapped a major locus between markers Xgwm210 and Xbarc1138.2 explained 32.7, 21.6, and $16.4 \%$ of MRL phenotypic variations under $\mathrm{CK}$, low $\mathrm{N}$ and low $\mathrm{P}$ conditions, respectively (Figure 1; Table 2). In fact, this locus has been reported controlling multi-root morphologic parameters (Ren et al., 2012) and yield components (Hai et al., 2008). The constitutively expression under different $\mathrm{N}$ and $\mathrm{P}$ supply levels in this research and its association with QTL for grain yield components suggested the importance of this locus in improving wheat nutrients use efficiency and yield.

Among the 13 loci for root traits detected in the current study, three loci ( $q R D W . L N-4 D, q R D W . L N-6 A$, and $q M R L . L N-$ $5 A)$ specifically expressed under low $\mathrm{N}$ condition and two loci ( $q M R L . L P-5 A$ and $q M R L . L P-6 B$ ) specifically expressed under low $\mathrm{P}$ condition (Figure 1). $q R D W . L N-6 A$ specifically expressed under low $\mathrm{N}$ condition and coincided with several previously reported QTLs for KNS (Kernel number per spike), HI (Harvest index) and TKW (Thousand kernel weight) under low $\mathrm{N}$ condition. qMRL.LP-5A specifically expressed under low $\mathrm{P}$ condition and coincided with previously reported QTLs for DM (Dry matter) under CK and low P condition (Xu et al., 2014). qMRL.LP-6B was tightly linked with previously reported QTLs for root length (Maccaferri et al., 2016; Iannucci et al., 2017), indicating the sustainable expression in different genetic background. These loci might be useful in improving the adaptability of root system to low nutrient availability.

In summary, this work detected 17 QTLs for root traits located at 13 loci on 11 chromosomes under different N and P Supply

\section{REFERENCES}

An, D. G., Su, J. Y., Liu, Q. Y., Li, B., Jing, R. L., Li, J. Y., et al. (2006). Mapping QTLs for nitrogen uptake in relation to the early growth of wheat (Triticum aestivum L.). Plant Soil 284, 73-84. doi: 10.1007/s11104-006-0030-3

Atkinson, J. A., Wingen, L. U., Griffiths, M., Pound, M. P., Gaju, O., Foulkes, M. J., et al. (2015). Phenotyping pipeline reveals major seedling root growth QTL in hexaploid wheat. J. Exp. Bot. 66, 2283-2292. doi: 10.1093/jxb/erv006

Basten, C. J., Weir, B. S., and Zeng, Z. B. (2001). QTL CARTOGRAPHER, Version 1.15. Raleigh, NC: Department of Statistics, North Carolina State University.

Börner, A., Plaschke, J., Korzun, V., and Worland, A. J. (1996). The relationships between the dwarfing genes of wheat and rye. Euphytica 89, 69-75. doi: 10.1007/BF00015721 levels. Encouragingly, we found that QTL analysis of root traits based on hydroponic culture can provide valuable clues for the development of wheat varieties with large and deep root system through QTL pyramiding. Considering the complexity of the genetic basis of root traits, more studies are needed to further validate these regions as targets for genetic improvement of root traits in field conditions.

\section{AUTHOR CONTRIBUTIONS}

YR and YQ performed the experiments; DL and AZ were responsible for DNA markers analysis and linkage analysis; YR and YX contributed to genetic mapping and QTL analysis; XZ, YT, and CZ conceived and designed the experiments; YR and YT wrote the paper.

\section{ACKNOWLEDGMENTS}

This research was supported by the National Key Research and Development Program of China (2016YFD0300205), the National Natural Science Foundation of China (31201678, 31572197) and Natural Science Foundation of Henan province (162300410133).

\section{SUPPLEMENTARY MATERIAL}

The Supplementary Material for this article can be found online at: https://www.frontiersin.org/articles/10.3389/fpls.2017. 02096/full\#supplementary-material

Figure S1 | The hydroponic equipment utilized in this work. The length, width and height of the hydroponic equipment are $2.5,1.5$, and $0.5 \mathrm{~m}$, respectively.

Figure S2 | QTLs for the investigated root traits, tiller number, shoot dry weight, nitrogen, and phosphorus uptake under $\mathrm{CK}$, low $\mathrm{N}$ and low $\mathrm{P}$ conditions.

Table S1 | The values of root dry weight and maximum root length of the recombinant inbred lines under $\mathrm{CK}$, low $\mathrm{N}$, and low $\mathrm{P}$ conditions.

Table S2 | Mean values and ranges for tiller number, shoot dry weight, nitrogen and phosphorus uptake in the RIL population and their parents under CK, low N, and low $\mathrm{P}$ conditions.

Table S3 | Correlation coefficients between the traits under CK, low N, and low P conditions.

Table S4 | Pyramiding QTLs for root biomass detected under low nitrogen condition enhanced $\mathrm{N}$ and $\mathrm{P}$ uptake.

Table S5 | Detected QTLs for tiller number, shoot dry weight, nitrogen and phosphorus uptake.

Canè, M. A., Maccaferri, M., Nazemi, G., Salvi, S., Francia, R., Colalongo, C., et al. (2014). Association mapping for root architectural traits in durum wheat seedlings as related to agronomic performance. Mol Breed. 34, 1629-1645. doi: 10.1007/s11032-014-0177-1

de Dorlodot, S., Forster, B., Pagès, L., Price, A., Tuberosa, R., and Draye, X. (2007). Root system architecture: opportunities and constraints for genetic improvement of crops. Trends Plant Sci. 12, 474-481. doi: 10.1016/j.tplants.2007.08.012

Den Herder, G., Van Isterdael, G., Beeckman, T., and De Smet, I. (2010). The roots of a new green revolution. Trends Plant Sci. 15, 600-607. doi: 10.1016/j.tplants.2010.08.009

Ehdaie, B., Merhaut, D. J., Ahmadian, S., Hoops, A. C., Khuong, T., Layne, A. P., et al. (2010). Root system size influences water-nutrient uptake 
and nitrate leaching potential in wheat. J. Agron. Crop Sci. 196, 455-466. doi: 10.1111/j.1439-037X.2010.00433.x

Guo, J. H., Liu, X. J., Zhang, Y., Shen, J. L., Han, W. X., Zhang, W. F., et al. (2010). Significant acidification in major Chinese croplands. Science 327, 1008-1010. doi: $10.1126 /$ science. 1182570

Hai, L., Guo, H. J., Wagner, C., Xiao, S. H., and Friedt, W. (2008). Genomic regions for yield and yield parameters in Chinese winter wheat (Triticum aestivum L.) genotypes tested under varying environments correspond to QTL in widely different wheat materials. Plant Sci. 175, 226-232. doi: 10.1016/j.plantsci.2008.03.006

Hochholdinger, F., and Tuberosa, R. (2009). Genetic and genomic dissection of maize root development and architecture. Curr. Opin. Plant Biol. 12, 172-177. doi: 10.1016/j.pbi.2008.12.002.

Holford, I. C. R. (1997). Soil phosphorus: its measurement, and its uptake by plants. Aust. J. Soil Res. 35, 227-239. doi: 10.1071/S96047

Horn, R., Wingen, L. U., Snape, J. W., and Dolan, L. (2016). Mapping of quantitative trait loci for root hair length in wheat identifies loci that co-locate withloci for yield compone-nts. J. Exp. Bot. 67, 4535-4543. doi: $10.1093 /$ jxb/erw228.

Iannucci, A., Marone, D., Russo, M. A., De Vita, P., Miullo, V., Ferragonio, P., et al. (2017). Mapping QTL for root and shoot morphological traits in a Durum Wheat $\times$ T. dicoccum segregating population at seedling stage. Int. J. Genomics 2017:6876393. doi: 10.1155/2017/6876393

Laperche, A., Devienne-Barret, F., Maury, O., Le Gouis, J., and Ney, B. (2006). A simplified conceptual model of carbon/nitrogen functioning for QTL analysis of winter wheat adaptation to nitrogen deficiency. Theor. Appl. Genet. 113, 1131-1146. doi: 10.1007/s00122-006-0373-4

Li, X. P., Zhao, X. Q., He, X., Zhao, G. Y., Li, B., Liu, D. C., et al. (2011). Haplotype analysis of the genes encoding glutamine synthetase plastic isoforms and their association with nitrogen-use- and yield-related traits in bread wheat. New Phytol. 189, 449-458. doi: 10.1111/j.1469-8137.2010.03490.x

Liang, Q., Cheng, X. H., Mei, M. T., Yan, X. L., and Liao, H. (2010). QTL analysis of root traits as related to phosphorus efficiency in soybean. Ann. Bot. 106, 223-234. doi: 10.1093/aob/mcq097

Liao, M., Fillery, I. R. P., and Palta, J. A. (2004). Early vigorous growth is a major factor influencing nitrogen uptake in wheat. Funct. Plant Biol. 31, 121-129. doi: 10.1071/FP03060

Lynch, J. (2007). Roots of the second green revolution. Aust. J. Bot. 55, 493-512. doi: 10.1071/BT06118

Maccaferri, M., EI-Feki, W., Nazemi, G., Salvi, S.,Canè, M. A., Colalongo, M. C., et al. (2016). Prioritizing quantitative trait loci for root system architecture in tetraploid wheat. J. Exp. Bot. 67, 1161-1178. doi: 10.1093/jxb/erw039

Quraishi, U. M., Abrouk, M., Murat, F., Pont, C., Foucrier, S., Desmaizieres, G., et al. (2011). Cross-genome map based dissection of a nitrogen use efficiency ortho-metaQTL in bread wheat unravels concerted cereal genome evolution. Plant J. 65, 745-756. doi: 10.1111/j.1365-313X.2010.04461.x

Ren, Y. Z., He, X., Liu, D. C., Li, J. J., Zhao, X. Q., Li, B., et al. (2012). Major quantitative trait loci for seminal root morphology of wheat seedlings. Mol. Breeding 30, 139-148. doi: 10.1007/s11032-011-9605-7

Ryan, P. R., Liao, M., Delhaize, E., Rebetzke, G. J., Weligama, C., Spielmeyer, W., et al. (2015). Early vigour improves phosphate uptake in wheat. J. Exp. Bot. 66, 7089-7100. doi: 10.1093/jxb/erv403

Sanguineti, M. C., Li, S., Maccaferri, M., Corneti, S., Rotondo, F., Chiari, T., et al. (2007). Genetic dissection of seminal root architecture in elite durum wheat germplasm. Ann. Appl. Biol. 151, 291-305. doi: 10.1111/j.1744-7348.2007.00198

Sharma, S., Xu, S. Z., Ehdaie, B., Hoops, A., Close, T. J., Lukaszewski, A. J., et al. (2011). Dissection of QTL effects for root traits using a chromosome armspecific mapping population in bread wheat. Theor. Appl. Genet. 122, 759-769. doi: 10.1007/s00122-010-1484-5

Steele, K. A., Virk, D. S., Kumar, R., Prasad, S. C., and Witcombe, J. R. (2007). Field evaluation of upland rice lines selected for QTLs controlling root traits. Field Crop Res. 101, 180-186. doi: 10.1016/j.fcr.2006.11.002
Su, J. Y., Xiao, Y. M., Li, M., Liu, Q. Y., Li, B., Tong, Y. P., et al. (2006). Mapping QTLs for phosphorus-deficiency tolerance at wheat seedling stage. Plant Soil 281, 25-36. doi: 10.1007/s11104-005-3771-5

Su, J. Y., Zheng, Q., Li, H. W., Li, B., Jing, R. L., Tong, Y. P., et al. (2009). Detection of QTLs for phosphorus use efficiency in relation to agronomic performance of wheat grown under phosphorus sufficient and limited conditions. Plant Sci. 176, 824-836. doi: 10.1016/j.plantsci.2009.03.006

Tuberosa, R., Sanguineti, M. C., Landi, P., Michela Giuliani, M., and Salvi, S. (2002). Identification of QTLs for root characteristics in maize grown in hydroponics and analysis of their overlap with QTLs for grain yield in the field at two water regimes. Plant Mol. Biol. 48, 697-712. doi: 10.1023/A:10148976 07670

Uga, Y., Sugimoto, K., Ogawa, S., Rane, J., Ishitani, M., Hara, N., et al. (2013). Control of root system architecture by DEEPER ROOTING 1 increases rice yield under drought conditions. Nat. Genet. 45, 1097-1102. doi: $10.1038 /$ srep 05563

Vance, C. P. (2001). Symbiotic nitrogen fixation and phosphorus acquisition: plant nutrition in a world of declining renewable resources. Plant Physiol. 127, 390-397. doi: 10.1104/pp.010331

Vance, C. P., Uhde-Stone, C., and Allan, D. L. (2003). Phosphorus acquisition and use: critical adaptations by plants for securing a nonrenewable resource. New Phyto. 157, 423-447. doi: 10.1046/j.1469-8137.2003.00695.x

Waines, J. G., and Ehdaie, B. (2007). Domestication and crop physiology: roots of Green-Revolution wheat. Ann. Bot. 100, 991-998. doi: 10.1093/aob/mcm 180

Wang, Y., Thorup-Kristensen, K., Jensen, L. S., and Magid, J. (2016). Vigorous root growth isa better indicator of early nutrient uptake than root hair traits in spring wheat grown un-der low fertility. Front. Plant Sci. 7:865. doi: $10.3389 /$ fpls.2016.00865

Xie, Q., Fernando, K. M., Mayes, S., and Sparkes, D. L. (2017). Identifying seedling root architectural traits associated with yield and yield components in wheat. Ann. Bot. 119, 1115-1129. doi: 10.1093/aob/mcx001.

Xu, Y. F., An, D. G., Liu, D. C., Zhang, A. M., Xu, H. X., and Li, B. (2012) Mapping QTLs with epistatic effects and QTL $\times$ treatment interactions for salt tolerance at s-eedling stage of wheat. Euphytica 186, 233-245. doi: 10.1007/s10681-012-0647-7

Xu, Y. F., Wang, R. F., Tong, Y. P., Zhao, H. T., Xie, Q. G., Liu, D. C., et al. (2014). Mapping QTLs for yield and nitrogen-related traits in wheat: influence of nitrogen and phosphorus fertilization on QTL expression. Theor. Appl. Genet. 127, 59-72. doi: 10.1007/s00122-013-2201-y

Yuan, Y. Y., Gao, M. G., Zhang, M. X., Zheng, H. H., Zhou, X. W., Guo, Y., et al. (2017). QTL mapping for phosphorus efficiency and morphological traits at seedling and mature-ty stages in wheat. Front. Plant Sci. 8:614. doi: $10.3389 /$ fpls.2017.00614

Zadoks, J. C., Chang, T. T., and Konzak, C. F. (1974). A decimal code for the growth stages of cereals. Weed Res. 14, 415-421.

Zeng, Z. B. (1994). Precision mapping of quantitative trait loci. Genetics 136, 1457-1468.

Zhang, J., Ju, X. T., Gao, Q., and Zhang, F. S. (2005). Recovery of labeled nitrate-N in different soil layers by two crops, spinach and wheat. Sci. Agricult. Sin. 38, 333-340.

Conflict of Interest Statement: The authors declare that the research was conducted in the absence of any commercial or financial relationships that could be construed as a potential conflict of interest.

Copyright (c) 2017 Ren, Qian, Xu, Zou, Liu, Zhao, Zhang and Tong. This is an open-access article distributed under the terms of the Creative Commons Attribution License (CC BY). The use, distribution or reproduction in other forums is permitted, provided the original author(s) or licensor are credited and that the original publication in this journal is cited, in accordance with accepted academic practice. No use, distribution or reproduction is permitted which does not comply with these terms. 